\title{
Serum Metabolomic Profiling Reveals Potential Biomarkers in Assessing The Management of Women with Polycystic Ovary Syndrome: A Randomized Controlled Trial
}

\section{Xuesong Ding}

Chinese Academy of Medical Sciences and Peking Union Medical College

\section{Yan Deng}

Chinese Academy of Medical Sciences and Peking Union Medical College

\section{Yan-fang Wang}

Chinese Academy of Medical Sciences and Peking Union Medical College

\section{Wei Xue}

Chinese Academy of Medical Sciences and Peking Union Medical College Shi-yang Zhu

Chinese Academy of Medical Sciences and Peking Union Medical College

\section{Xiao Ma}

Chinese Academy of Medical Sciences and Peking Union Medical College

\section{Ruilin Ma}

Chinese Academy of Medical Sciences and Peking Union Medical College

Ai-jun Sun ( $\nabla$ saj_pumch@sina.com )

Chinese Academy of Medical Sciences and Peking Union Medical College https://orcid.org/0000-00020049-0906

\section{Research article}

Keywords: polycystic ovary syndrome, metabolomic approach, management evaluation

Posted Date: July 15th, 2020

DOl: https://doi.org/10.21203/rs.3.rs-38668/v1

License: (c) (1) This work is licensed under a Creative Commons Attribution 4.0 International License. Read Full License 
Version of Record: A version of this preprint was published at Chinese Medical Journal on December 6th, 2021. See the published version at https://doi.org/10.1097/CM9.0000000000001705. 


\section{Abstract}

Background: As one of the most common endocrinal disorder for the women at childbearing age, the diagnostic criteria of polycystic ovary syndrome (PCOS) have been defined distinctly among different international health organizations. The diverse manifestations and heterogenic etiology of PCOS also bring about difficulties for its diagnosis and management assessment. Though the elevated androgen level is acknowledged to be the most obvious biomarker for PCOS diagnosis and management evaluation, it is not always present in patients and the mechanism of hyperandrogenism has not been fully elucidated. Therefore, more efficient biomarkers representing progression of PCOS are expected to be integrated into monitoring the management process using metabolomic approach.

Methods: In this prospective randomized controlled trial, classical diagnostic parameters, blood glucose, and metabolome were measured in 123 PCOS patients simultaneously before and at 2 and 3 months of different medical interventions. The metabolite fingerprints were detected filtered out by comparing data at baseline and 3 months based on multivariate statistical analysis, followed by validation with receiver operating characteristic (ROC) curve on data collected at 2 months.

Results: A set of metabolites including glutamic acid, aspartic acid, 1-methylnicotinamide, acetylcarnitine, glycerophosphocholine, and oleamide, were filtered out with high performance in representing the improvement through three-month management of PCOS with high sensitivity and specificity in ROC analysis.

Conclusions: The 6 metabolites were representative for the remission of PCOS through medical intervention, making them a set of potential biomarkers on assessing the outcome of PCOS management.

Trial registration: ClinicalTrials.gov, NCT03264638. Registered 29 August 2017 - Retrospectively registered, https://clinicaltrials.gov/ct2/show/NCT03264638

\section{Background}

PCOS is one of the most common diseases among women of childbearing age, which is also one of the major causes of infertility due to the malfunction of ovulation, a manifestation of the disrupted reproductive endocrine system. However, the heterogenic etiology and diversified symptom and sign of PCOS bring about a large amount of difficulties in diagnosis and management [1]. Meanwhile, the diagnostic criteria of PCOS have undergone many alternations by different international health organizations, resulting in the fluctuation of its prevalence, ranging from 8-13\% [2]. The most obvious biomarker, evaluated androgen, does not appear in every case. PCOS has been the hot spot in reproductive endocriology research field for years, but the knowledge of its pathogenesis and related treatments are still very limited. Though genome-wide assiociation studies have revealed the genetic foundition of PCOS, the connection from gene to phenotypes has yet been delineated. Therefore, 
additional parameters are expected to be recruited to assess the progression and management outcomes of PCOS.

Metabolome refers to the collection of small metabolites within cells, tissues, and biofluids which can directly reflect the micro-environment under the influence of genetic regulation and external exposure [4]. As the end product of genome and transcriptome, metabolome has been recognized as better representatives, closely reflecting the actual phenotype, as result of both external environment changes and inherited material.The advancement of Liquid chromatography-mass spectrometry/ mass spectrometry (LC-MS/MS) allows researchers to evaluate all the metabolites non-invasively in a hypothesis-free way with great accuracy and efficiency $[5,6]$.

Besides ovulation disorder, PCOS has been closely related to alternated metabolism, there was an increased incidence of developing obese and impaired insulin sensitivity in PCOS population, accompanied with higher risk of cardiovasculor disease and type 2 diabetes as long-term complications. Metabolome has been extensively studied to evaluate the process of endocrinological disorders such as insulin resistance and type 2 diabetes mellitus, whereas the metabolic profile in PCOS needs further excavation and more refined analysis $[7,8]$. To provide new insights on the association between metabolomic profiles and PCOS progression or remission, we characterized the metabolism fingerprint associated with PCOS by applying a LC-MS/MS metabolomic approach to serum samples obtained from three differently intervention groups at baseline, 2nd month, and 3rd month, and non-targeted multivariate statistical analysis was performed to filter out the most relavent candidate for potential biomarker.

\section{Methods}

\section{Study design and participants}

This was a single center, prospective, open-label, parallel-group, randomized clinical trial (figure 1). Baseline and follow-up examinations were conducted at the clinics of Peking Union Medical College Hospital (PUMCH) in Beijing, China, from December 2016 to September 2017. Participants were recruited by advertisement. Women aged 18-39 years and diagnosed with PCOS based on Rotterdam consensus (two of three criteria: oligo-/anovulation, hyperandrogenemia, and sonographic polycystic ovary morphology) were eligible. Diagnosis of oligo-/anovulation was based on a menstrual pattern of oligo-/amenorrhoea (cycle >35 days) and/or a low mid-luteal serum progesterone concentration. Hyperandrogenemia was diagnosed either clinically (acne/hirsutism) or biochemically (testosterone $\geq 0.75 \mathrm{ng} / \mathrm{ml}$ ). Polycystic ovaries were $\geq 12$ follicles $(2-9 \mathrm{~mm}$ ) and/or an ovarian volume of $>10 \mathrm{ml}$ under ultrasonography. Exclusion criteria were medical or surgical treatment of PCOS within 3 months, thyroid disease, hyperprolactinemia, active liver disease, history of cardiac or renal failure, hormone medication, alcohol use, and regular smoking. This trial was approved by the Ethics Committee of Peking Union Medical College Hospital, Peking Union Medical College, Chinese Academy of Medical Science (No. ZS-1222, dated November 29, 2016). All participants provided written informed consent.

\section{Interventions}


Participants were randomly assigned to one of three intervention groups in 1:1:1 ratio by researchers. The random numbers were generated by computer and assigned to each eligible patients uniquely. All drugs were taken orally for 21 consecutive days followed by 7 days of drug-free. Group A took $7 \mathrm{~g}$ Dingkundan daily; group B took one tablet of Diane-35 daily; group $\mathrm{C}$ took both $7 \mathrm{~g}$ Dingkundan and one tablet of Diane-35 daily. The intervention lasts for three months. Physical examination (body mass index), clinical biochemical (total testosterone, fasting glucose, glucose $1 \mathrm{~h}$, glucose $2 \mathrm{~h}, \mathrm{HbA1c}$, fasting insulin , insulin $0.5 \mathrm{~h}$, insulin $1 \mathrm{~h}$, insulin $2 \mathrm{~h}$, total cholesterol, triglyceride, high-density lipoprotein cholesterol, low-density lipoprotein ), and metabolomic analysis were performed at baseline, two-months, and three-month treatment as the primary outcomes.

\section{Clinical biochemical analysis}

Measurements of testosterone ( $T$ ), sulfated-dehydroepiandrosterone (DHEAS) were performed by chemiluminescence immunoassay (CLIA) using Beckman Coulter DXI 800.

\section{Metabolite extraction}

High Performance Liquid Chromatography (HPLC) grade methanol was pre-chilled at $-80^{\circ} \mathrm{C}$ before use. The pre-chilled methanol was added to $100 \mu \mathrm{L}$ serum samples up to $80 \%(\mathrm{v} / \mathrm{v})$. Samples were placed at $-80^{\circ} \mathrm{C}$ for 1 hour and then centrifuged at $14000 \mathrm{~g}$ for $30 \mathrm{~min}$. Supernatants were carefully transferred to new Eppendorf tubes. Subsequently, all supernatants were dried and stored in $-80^{\circ} \mathrm{C}$ freezer for future analysis.

\section{Metabolomics analysis}

Samples were re-dissolved using $60 \mu \mathrm{L}$ of $80 \%(\mathrm{v} / \mathrm{v})$ methanol. After centrifugation for 20 minutes, supernatants were transferred to sample vials for LC-MS/MS analysis. Metabolites were profiled using $Q$ Exactive mass spectrometer coupled with UHPLC system (Thermo, CA). In the analysis, data-dependent acquisition in positive and negative ion mode was performed. BEH Amide column $(2.1 \mathrm{~mm} \times 100 \mathrm{~mm})$ was used for LC separation with flowrate of $250 \mu \mathrm{L} / \mathrm{min}$. In positive ion mode, $95 \%$ and $50 \%$ of acetonitrile with $5 \mathrm{mM}$ ammonium formate and $0.1 \%$ formic acid was used as mobile phases. In negative ion mode, $95 \%$ and $50 \%$ of acetonitrile with $5 \mathrm{mM}$ ammonium acetate at $\mathrm{pH} 9.0$ (adjusted using ammonium hydroxide) were applied as mobile phase $A$ and B. electrospray ionization (ESI) voltages of $3.5 \mathrm{kV}$ in positive mode and $2.5 \mathrm{kV}$ in negative mode were used respectively. MS scan with 70,000 resolution and MS/MS scans of top 10 the most intense precursors with 17,500 resolution was applied. Mass range of $70-1050 \mathrm{~m} / \mathrm{z}$ was used to acquire data in positive ion mode and $80-1200 \mathrm{~m} / \mathrm{z}$ for negative mode was used. $10 \mu \mathrm{L}$ of serum from each of all the samples was mixed for the sample quality control (QCs) to assess the repeatability of samples and validating the stability of the whole analysis. Before the experiment, five QC samples were successively tested for balancing the columns, and subsequently, 1 QC sample was acquired for every 15 samples to further monitor the stability of the LCMS/MS analysis. 


\section{Metabolite identification}

Metabolites were identified using Tracerfinder 3.2 (Thermo Fisher, CA) based on in-house MS/MS library. The in-house MS/MS library was established using either chemical standards or biological samples. Two levels of metabolite identifications were achieved, one confirmed by MS/MS and the rest matched using precursor mass. Therefore, the ones without MS/MS confirmation were assigned as metabolite candidates. For metabolites having MS/MS confirmation, only the ones with LS score above 30 were considered as confidently identified. Otherwise, metabolites had only tentative assignments. In-house software "Metalnt" was incorporated for high throughput data analysis.

\section{Statistical and pathway analysis}

Metabolites were normalized by the sum of area of identified peaks before subsequent statistical analysis. Orthogonal partial least squares discriminate analysis (OPLSDA) were performed using SIMCA 14 (Umetrics, Sweden). Student's t-test results and FDR adjustment were acquired from Metaboanalyst 3.0 (www.metaboanalyst.ca). The Human Metabolome Database (www.HMDB.ca), The Small Molecule Pathway Database (SMPDB), and Metaboanalyst (www.metaboanalyst.ca) were used for pathway analysis.

\section{Results}

\section{Comparison of clinical characteristics and biochemical data at baseline}

The patients recruited for this study were diagnosed based on the Rotterdam diagnostic criteria (two of oligo- or anovulation, clinical and/or biochemical hyperandrogenism, or polycystic morphological changes on ultrasound, after exclusion of other relevant diseases). Their baseline characters of three groups was summarized in Table 1, including the classic clinic laboratory results relevant to PCOS and metabolic status of patients, for instance, the diagnostic criteria for chemical hyperandrogenism (total testosterone and DHEAS), body mass index (BMI), blood glucose and insulin level through 2-hour oral glucose tolerance test (OGTT), and other parameters describing lipid metabolism (LDL-C, HDL-C, TC, and TG). As expected, no statistical difference was found in all listed parameters among three randomly assigned PCOS patient groups. 
Table 1

Demographic and Clinical Characteristics of the Participants at Baseline

\begin{tabular}{|c|c|c|c|c|}
\hline & $\begin{array}{l}\text { Group A } \\
(n=40)\end{array}$ & $\begin{array}{l}\text { Group B } \\
(n=42)\end{array}$ & $\begin{array}{l}\text { Group C } \\
(n=41)\end{array}$ & $P^{*}$ \\
\hline Age (year) & $27.5 \pm 3.4$ & $27.2 \pm 3.5$ & $26.7 \pm 6.4$ & 0.783 \\
\hline $\mathrm{BMI}\left(\mathrm{kg} / \mathrm{m}^{2}\right)$ & $26.1 \pm 5.4$ & $25.5 \pm 5.8$ & $26.4 \pm 5.9$ & 0.764 \\
\hline Total T (ng/mL) & $0.81 \pm 0.27$ & $0.72 \pm 0.20$ & $0.74 \pm 0.24$ & 0.220 \\
\hline Fasting glucose (mmol/L) & $5.1 \pm 0.4$ & $5.1 \pm 0.5$ & $5.2 \pm 0.7$ & 0.570 \\
\hline glucose 1 h (mmol/L) & $7.9 \pm 2.1$ & $8.2 \pm 3.0$ & $8.0 \pm 2.5$ & 0.866 \\
\hline glucose $2 \mathrm{~h}(\mathrm{mmol} / \mathrm{L})$ & $6.6 \pm 1.3$ & $7.0 \pm 2.3$ & $7.0 \pm 2.4$ & 0.700 \\
\hline HbA1c (\%) & $5.3 \pm 0.3$ & $5.3 \pm 0.4$ & $5.2 \pm 0.4$ & 0.596 \\
\hline Fasting Insulin $(\mu \mathrm{IU} / \mathrm{mL})$ & $16.32 \pm 8.77$ & $18.03 \pm 15.82$ & $18.78 \pm 13.12$ & 0.703 \\
\hline Insulin0.5 h( $\mu \mathrm{lU} / \mathrm{mL})$ & $128.00 \pm 64.03$ & $133.05 \pm 95.38$ & $112.01 \pm 60.05$ & 0.451 \\
\hline Insulin 1 h( $\mu \mathrm{lU} / \mathrm{mL})$ & $115.70 \pm 67.07$ & $124.20 \pm 74.41$ & $114.82 \pm 76.31$ & 0.860 \\
\hline Insulin 2 h( $\mu \mathrm{lU} / \mathrm{mL})$ & $94.38 \pm 61.67$ & $90.93 \pm 77.96$ & $75.869 \pm 68.93$ & 0.473 \\
\hline $\mathrm{TC}(\mathrm{mmol} / \mathrm{L})$ & $4.66 \pm 1.19$ & $4.65 \pm 0.92$ & $4.49 \pm 0.99$ & 0.731 \\
\hline $\mathrm{TG}(\mathrm{mmol} / \mathrm{L})$ & $3.18 \pm 12.51$ & $1.34 \pm 1.03$ & $1.36 \pm 0.99$ & 0.460 \\
\hline $\mathrm{HDL}-\mathrm{C}(\mathrm{mmol} / \mathrm{L})$ & $1.48 \pm 0.57$ & $1.31 \pm 0.33$ & $1.37 \pm 0.72$ & 0.463 \\
\hline LDL-C(mmol/L) & $2.90 \pm 0.84$ & $2.81 \pm 0.16$ & $2.78 \pm 0.75$ & 0.821 \\
\hline \multicolumn{5}{|c|}{$\begin{array}{l}\text { Each value represents mean } \pm \text { SEM; Group A: Dingkundan; Group B: Diane-35; Group C: Dingkundan } \\
\text { combined with Diane-35; *Between groups determined by one-way ANOVA. BMI: body mass index; } \\
\text { Total T: total testosterone; TC: total cholesterol; TG: triglyceride; HbA1c: hemoglobin A1c; HDL-C: High- } \\
\text { density lipoprotein cholesterol; and LDL-C: Low-density lipoprotein cholesterol. }\end{array}$} \\
\hline
\end{tabular}

\section{Improvement of classic clinical parameters between baseline and end of 3-month management.}

After a 3-month management using Dingkundan, Diane-35, and Dingkundan combined with Diane-35, symptom of irregular menstruation was corrected with the improvement of ovulation in all three groups. Information of classic clinical parameters and relavent metabolism characters applied to assess the improvement of PCOS status was collected in Table 2, describing the net changes of factors listed in baseline, with the asterisk labelling the significant difference between baseline and three-month data. In group B, with Diane-35, one of the conventional treatment of PCOS, the results showed a significant decrease in total testosterone levels compared with Dingkundan group, indicating an improvement of high androgen status after three-month management. Compared with group B, the combo of TCM Dingkundan and combined oral contraceptive pills Diane-35 (Group C) showed an additional effect on 
BMI reduction. However, for lipid metabolism, there were significant increases in total cholesterol (TC) and High-density lipoprotein cholesterol (HDL-C) levels in Group B and Group C, which diverted from the trend observed in Group A.

Table 2

Absolute Changes of Tested Parameters from Baseline to 3 Months

\begin{tabular}{|c|c|c|c|c|}
\hline & $\begin{array}{l}\text { Group A } \\
(n=40)\end{array}$ & $\begin{array}{l}\text { Group B } \\
(n=42)\end{array}$ & $\begin{array}{l}\text { Group C } \\
(n=41)\end{array}$ & $\mathrm{P}^{* *}$ \\
\hline $\operatorname{BMI}\left(\mathrm{kg} / \mathrm{m}^{2}\right)$ & $0.1(0.9)$ & $-0.0(1.2)$ & $-0.3(0.9)^{\star}$ & 0.242 \\
\hline Total T(ng/mL) & $-0.04(0.24)$ & $-0.14(0.16)^{\star}$ & $-0.14(0.17)^{\star}$ & 0.033 \\
\hline LH(IU/L) & $-0.10(5.33)$ & $-4.93(6.18)^{\star}$ & $-5.23(6.30)^{\star}$ & 0.01 \\
\hline $\mathrm{FSH}(\mathrm{IU} / \mathrm{L})$ & $-0.03(1.73)$ & $0.45(2.64)$ & $0.77(2.58)$ & 0.348 \\
\hline Fasting glucose( $\mathrm{mmol} / \mathrm{L})$ & $-0.1(0.3)^{\star}$ & $-0.3(0.3)^{\star}$ & $-0.2(0.5) \star$ & 0.139 \\
\hline Fasting insulin $(\mu \mathrm{IU} / \mathrm{mL})$ & $-0.77(6.91)$ & $-0.95(12.33)$ & $-3.39(11.79)$ & 0.492 \\
\hline 2 h-OGTT glucose(mmol/L) & $-0.4(1.3)$ & $0.3(1.8)$ & $-0.03(1.5)$ & 0.169 \\
\hline 2 h-OGTT insulin $(\mu \mathrm{IU} / \mathrm{mL})$ & $-8.12(79.91)$ & $2.09(60.92)$ & $6.40(48.35)$ & 0.592 \\
\hline $\mathrm{TC}(\mathrm{mmol} / \mathrm{l})$ & $-0.27(0.52) \star$ & $0.22(0.81)$ & $0.45(0.78)^{\star}$ & 0.000 \\
\hline $\mathrm{TG}(\mathrm{mmol} / \mathrm{l})$ & $0.28(0.59)^{\star}$ & $0.62(0.86)^{\star}$ & $0.42(1.04)^{\star}$ & 0.245 \\
\hline LDL-C(mmol/l) & $-0.25(0.40) \star$ & $-0.25(0.65)^{\star}$ & $-0.01(0.71)$ & 0.143 \\
\hline $\mathrm{HDL}-\mathrm{C}(\mathrm{mmol} / \mathrm{l})$ & $-0.18(0.56)$ & $0.35(0.50)^{*}$ & $0.24(0.83)$ & 0.002 \\
\hline
\end{tabular}

Each value represents mean (SEM); Group A: Dingkundan; Group B: Diane-35; Group C: Dingkundan combined with Diane-35; ${ }^{*}$ Between treatment groups determined by one-way ANOVA; ${ }^{*} \mathrm{P}<0.05$ for the comparison between baseline and 3 months. BMI: body mass index; TC: total cholesterol; TG: triglyceride; HbA1c: hemoglobin A1c; HDL-C: High-density lipoprotein cholesterol; and LDL-C: Low-density lipoprotein cholesterol.

\section{Metabolic pathway enrichment using metabolomic profile in PCOS patients before and after management}

In this research, more than 600 small molecules in blood serum were detected using LC-MS/MS and nontargeted analysis was performed, in which a total of 93 metabolites sharing consistent changes in all three treatment groups (VIP $>1$ in OPLSDA analysis in each medication group, FDR $<0.05$ before and after treatment). The abundance of these metabolites was normalization [Additional file 1]. Pathways were enriched using Metaboanalyst, and the metabolite sets enrichment were plotted in Fig. 2. The most 
significantly different pathways after three-month treatment were mainly related to amino acid metabolism, especially for one participant, aspartate.

\section{A set of 6 metabolites were filtered out through analysis}

From all the metabolites detected in patient serum, 6 metabolites were filtered out on the principles of high abundance, fold change $>1.5$ or $<0.75$, FDR $<0.0001$, and pathway related compounds, namely, glutamic acid, aspartic acid, 1-methylnicotinamide, acetylcarnitine, glycerophosphocholine, and oleamide. It turned out that most relavent pathways were related to amino acid metabolism. The outcomes showed that aspartate, nicotinate, and nicotinamide metabolism were enriched significantly after treatment for 3 months. The serum concentration of these 6 metabolites was plotted separately (Fig. 3). Aspartic acid, glycerophosphocholine, and oleamide showed a gradually increasing trend during management, whereas methylnicothinamide, L-acetylcarnitine, and glutamic acid decreased after treatment.

\section{Assessing management efficiency using these combinational biomarkers.}

The metabolites including glutamic acid, aspartic acid, 1-methylnicotinamide, acetylcarnitine, glycerophosphocholine, and oleamide were combined to build ROC curve to evaluate the status of baseline characters for group A, group B, group $C$, and group A plus $B$, based on their blood concentrations of group $\mathrm{C}$ after three-month management, and the results were plotted in Fig. 4 . All three groups were considered as remission since there was significant improvement on biochemical hyperandrogenism. Data of this set of metabolites from group $C$ were regarded as the reference to assess the outcome of these 3 groups, the area under the curve (AUC) for the other three circumstances were above 0.96 , suggesting these set of metabolites may be as efficient as blood testosterone level in monitoring the efficacy of PCOS management.

In addition, these 6 biomarkers have also been applied to assess the management efficiency of medical intervention at 2nd month of management. We applied these 6 biomarkers to re-evaluate the clinical progress at 2nd month of treatment using Dingkundan, Daine-35, and Dingkundan plus Daine-35, respectively (Fig. 5), from which the best drug efficiency has been seen in group $C$, suggesting the combo of herbal medicine and western drug were more efficient in treating PCOS than western drug alone.

\section{Discussion}

In the present study, these 6 metabolites were filtered out based on their significant and consistent change for 3 months treatment. They are equal to serum concentration alternation of testosterone for reflecting the progression of PCOS management.Their performance in assessing the efficiency was demonstrated using data collected from 2nd month.

In these 6 metabolites, two of them are amino acid. Aspartic acid is a non-essential amino acid involved in the synthesis of other amino acids, for example, asparagine and arginine. It also participates in tricarboxylic acid (TCA) cycle and urea cycles [9]. Aspartic acid also serves an important role in Malateaspartate shuttle, in which the electron generated from glycolysis is translocated cross the 
semipermeable mitochondrial inner membrane [10]. Notably, Aspartic acid also has a role in neural signal transfer [11]. The other non-essential amino acid selected is glutaric acid, serving as an indispensable precursor for the biosynthesis of gamma-aminobutyric acid (GABA) in neurons [12]. It also serves as the amino acid donor in ketoglutarate synthesis, an intermediate in the TCA cycle, providing pyruvate for further oxidation within mitochondria [13]. The changes of aspartic acid and glutaric acid illustrated an alternation in amino acid metabolism pattern of PCOS patients.

Methylnicotinamide is a member involved in nicotinamide metabolism pathway and mainly produced in liver with anti-inflammatory properties [14]. Methylnicotinamide was predicted to be the endogenous activator of prostacyclin, which involved in the physiological regulation of thrombogenesis and inflammatory process in cardiovascular system (CVS) [15]. The decrease of methylnicotinamide during treatment is in conformity with the expectation of reducing the long-term risk of complication in CVS.

In the metabolome data, several long-chain fatty acids were significantly decreased with lower level of several acyl-carnitines [16]. These evidences indicate a reduced lipid synthesis. On the other hand, increased glycerophosphocholine is observed, which means medication increases hydrolysis of phosphatidylcholine [17].

Interestingly, oleamide is found to be elevated by 3.7 and 4.2 folds after medication of herbal and western drug, respectively. The combined drug treatment even increased oleamide level by 6 folds. It has been known that oleamide induces sleep in animals. This may lead to a side effect in treatment [18].

As a complex syndrome, PCOS is a combination of endocrine disorder, reproductive malfunction, and metabolism abnormality. The diagnostic criteria of PCOS vary from institute due to its sophisticated etiology and diversified manifestation, causing a fluctuated prevalence in women at child-bearing age. For instance, PCOS was defined by European society of human reproduction and embryology (ESHRE) in Rotterdam as a combination of 2 out of 3 criteria, namely an irregular menstrual cycle, sign of hyperandrogenism, and polycystic ovary morphology (PCOM) [19]; whereas the androgen excess society (AES) focused more on androgen-excess, defining PCOS as clinical or biochemical hyper-androgen combined with oligo-/anovulation or PCOM [20].

The heterogenicity of etiology and complex clinical manifestations bring in large difficulty for the accurate diagnosis of patients at risk. Accompanied by endocrinological disorder, PCOS patients also show metabolic disorder, and insulin resistance is observed in $60-80 \%$ of PCOS patients [21]. Metabolomics approach has been successfully applied to investigate the pathogenesis and potential diagnostic biomarkers in other types of metabolic disorientation such as type II diabetes, cardiovascular diseases, and insulin resistance $[7,8,22]$. Thereby, using metabolomic approach to profile the fingerprint of PCOS metabolic alternation may provide insights into the potential diagnostic biomarkers.

In this way, we found 6 metabolites with great potential for assessing PCOS management process of medical treatment. However, analysis of metabolomics is a relatively complicated process which may 
lead to certain difficulty in its popularization, and further trial with larger sample size is expected to validate their effect before applying them in clinical practice.

\section{Conclusions}

With metabolomic approach, LC-MS/MS was applied to find out the consistent changes of certain metabolites, from which 6 metabolites were picked for their significant fold changes before and after treatment of PCOS for 3 months, namely, glutamic acid, aspartic acid, 1-methylnicotinamide, acetylcarnitine, glycerophosphocholine, and oleamide. Their high-fidelity property for monitoring the management process was proved using ROC analysis, with AUC no less than 0.96 through 4 groups, and it was applicable for evaluating the management efficiency in the 2 nd month data. Larger experiment setup is expected to further validate of these 6 metabolites' value in monitoring management efficiency in PCOS.

\section{List Of Abbreviations}




\begin{tabular}{|ll|}
\hline Abbreviation & full name \\
\hline PCOS & polycystic ovary syndrome \\
\hline ROC & receiver operating characteristic \\
\hline LC & Liquid chromatography \\
\hline MS & mass spectrometry \\
\hline DHEAS & Dehydroepiandrosterone sulfate \\
\hline BMI & Body Mass Index \\
\hline OGTT & oral glucose tolerance test \\
\hline VIP & visual infusion phlebitis \\
\hline OPLSDA & orthogonal partial least squares discriminant analysis \\
\hline FDR & False discovery rate \\
\hline AUC & area under the curve \\
\hline TCA & tricarboxylic acid \\
\hline GABA & gamma-aminobutyric acid \\
\hline CVS & cardiovascular system \\
\hline ESHRE & European society of human reproduction and embryology \\
\hline PCOM & polycystic ovary morphology \\
\hline AES & androgen excess society \\
\hline PUMCH & Peking Union Medical College Hospital \\
\hline
\end{tabular}

\section{Declarations}

\section{Ethics approval and consent to participate}

This trial was approved by the Ethics Committee of Peking Union Medical College Hospital, Peking Union Medical College, Chinese Academy of Medical Science (No. ZS-1222, dated November 29, 2016). All participants provided written informed consent.

\section{Consent for publication}

Not applicable.

\section{Availability of data and materials}


The datasets used and/or analysed during the current study are available from the corresponding author on reasonable request.

\section{Competing interests}

The authors declare that they have no competing interests.

\section{Funding}

No external financial support was used.

\section{Authors' contributions}

AJS contributed to the conceptualization and design of the study, the data collection and interpretation. $X D$ wrote and editing the manuscript. YD and XD contributed to the protocol planning, data collection and analysis. WX, FY, RM, SZ, XM contributed to patients recruitment and data collection. All authors read and approved the final version of the paper.

\section{Acknowledgements}

We are grateful to all the women who participated in our study, and thank the Clinical Laboratory Department of Peking Union Medical College Hospital for their great help.

\section{References}

1. R. Azziz, E. Carmina, Z. Chen, A. Dunaif, J.S.E. Laven, R.S. Legro, D. Lizneva, B. Natterson-Horowtiz, H.J. Teede, B.O. Yildiz, Polycystic ovary syndrome., Nat. Rev. Dis. Prim. 2 (2016) 16057. https://doi.org/10.1038/nrdp.2016.57.

2. H.J. Teede, D. Ph, M.L. Misso, D. Ph, B.S. Hons, Recommendations from the international evidencebased guideline for the assessment and management of polycystic ovary syndrome, 110 (2018) 364-379.

3. J.J. Kim, Y.M. Choi, Phenotype and genotype of polycystic ovary syndrome in Asia: Ethnic differences., J. Obstet. Gynaecol. Res. (2019). https://doi.org/10.1111/jog.14132.

4. N. Zamboni, A. Saghatelian, G.J. Patti, Defining the metabolome: size, flux, and regulation., Mol. Cell. 58 (2015) 699-706. https://doi.org/10.1016/j.molcel.2015.04.021.

5. X. Lu, X. Zhao, C. Bai, C. Zhao, G. Lu, G. Xu, LC--MS-based metabonomics analysis, J. Chromatogr. B. $866(2008) 64-76$.

6. G. Theodoridis, H.G. Gika, I.D. Wilson, LC-MS-based methodology for global metabolite profiling in metabonomics/metabolomics, TrAC Trends Anal. Chem. 27 (2008) 251-260.

7. J.T. Brindle, H. Antti, E. Holmes, G. Tranter, J.K. Nicholson, H.W.L. Bethell, S. Clarke, P.M. Schofield, E. McKilligin, D.E. Mosedale, others, Rapid and noninvasive diagnosis of the presence and severity of coronary heart disease using 1 H-NMR-based metabonomics, Nat. Med. 8 (2002) 1439. 
8. X. Zhao, J. Fritsche, J. Wang, J. Chen, K. Rittig, P. Schmitt-Kopplin, A. Fritsche, H.-U. Häring, E.D. Schleicher, G. Xu, others, Metabonomic fingerprints of fasting plasma and spot urine reveal human pre-diabetic metabolic traits, Metabolomics. 6 (2010) 362-374.

9. J.R. Barrio, J.E. Egbert, E. Henze, H.R. Schelbert, F.J. Baumgartner, L-[4-11C] aspartic acid: enzymic synthesis, myocardial uptake, and metabolism, J. Med. Chem. 25 (1982) 93-96.

10. S.B. Digerness, W.J. Reddy, The malate-aspartate shuttle in heart mitochondria, J. Mol. Cell. Cardiol. 8 (1976) 779-785.

11. E.S. Levine, R.A. Crozier, I.B. Black, M.R. Plummer, Brain-derived neurotrophic factor modulates hippocampal synaptic transmission by increasing N-methyl-D-aspartic acid receptor activity, Proc. Natl. Acad. Sci. 95 (1998) 10235-10239.

12. T.T.F. Lima, J. Begnini, J. de Bastiani, D.B. Fialho, A. Jurach, M.C.P. Ribeiro, M. Wajner, C.F. de Mello, Pharmacological evidence for GABAergic and glutamatergic involvement in the convulsant and behavioral effects of glutaric acid, Brain Res. 802 (1998) 55-60.

13. C. Mühlhausen, B.C. Burckhardt, Y. Hagos, G. Burckhardt, B. Keyser, Z. Lukacs, K. Ullrich, T. Braulke, Membrane translocation of glutaric acid and its derivatives, J. Inherit. Metab. Dis. 31 (2008) 188193.

14. A. Jakubowski, M. Sternak, K. Jablonski, M. Ciszek-Lenda, J. Marcinkiewicz, S. Chlopicki, 1Methylnicotinamide protects against liver injury induced by concanavalin A via a prostacyclindependent mechanism: A possible involvement of IL-4 and TNF-\$\$Ș, Int. Immunopharmacol. 31 (2016) 98-104.

15. L. Mateuszuk, A. Jasztal, E. Maslak, M. Gasior-Glogowska, M. Baranska, B. Sitek, R. Kostogrys, A. Zakrzewska, A. Kij, M. Walczak, others, Antiatherosclerotic Effects of 1-Methylnicotinamide in Apolipoprotein E/Low-Density Lipoprotein Receptor--Deficient Mice: A Comparison with Nicotinic Acid, J. Pharmacol. Exp. Ther. 356 (2016) 514-524.

16. A.K. Hajra, J.E. Bishop, Glycerolipid biosynthesis in peroxisomes via the acyl dihydroxyacetone phosphate pathway, (1982).

17. K. Sonkar, V. Ayyappan, C.M. Tressler, O. Adelaja, R. Cai, M. Cheng, K. Glunde, Focus on the glycerophosphocholine pathway in choline phospholipid metabolism of cancer, NMR Biomed. (2019) e4112.

18. C.R. Hiley, P.M. Hoi, Oleamide: a fatty acid amide signaling molecule in the cardiovascular system?, Cardiovasc. Drug Rev. 25 (2007) 46-60.

19. E. Pages, Consensus on women's health aspects of polycystic ovary syndrome (PCOS), Hum Reprod. 27 (2012) 14-24.

20. N.F. Goodman, R.H. Cobin, W. Futterweit, J.S. Glueck, R.S. Legro, E. Carmina, American Association of Clinical Endocrinologists, American College of Endocrinology, and Androgen Excess and PCOS Society disease state clinical review: guide to the best practices in the evaluation and treatment of polycystic ovary syndrome-part 1, Endocr. Pract. 21 (2015) 1291-1300. 
21. E. Diamanti-Kandarakis, C.D. Christakou, Insulin resistance in PCOS, in: Diagnosis Manag. Polycystic Ovary Syndr., Springer, 2009: pp. 35-61.

22. Q. Huang, P. Yin, J. Wang, J. Chen, H. Kong, X. Lu, G. Xu, Method for liver tissue metabolic profiling study and its application in type 2 diabetic rats based on ultra performance liquid chromatographymass spectrometry, J. Chromatogr. B. 879 (2011) 961-967.

\section{Figures}

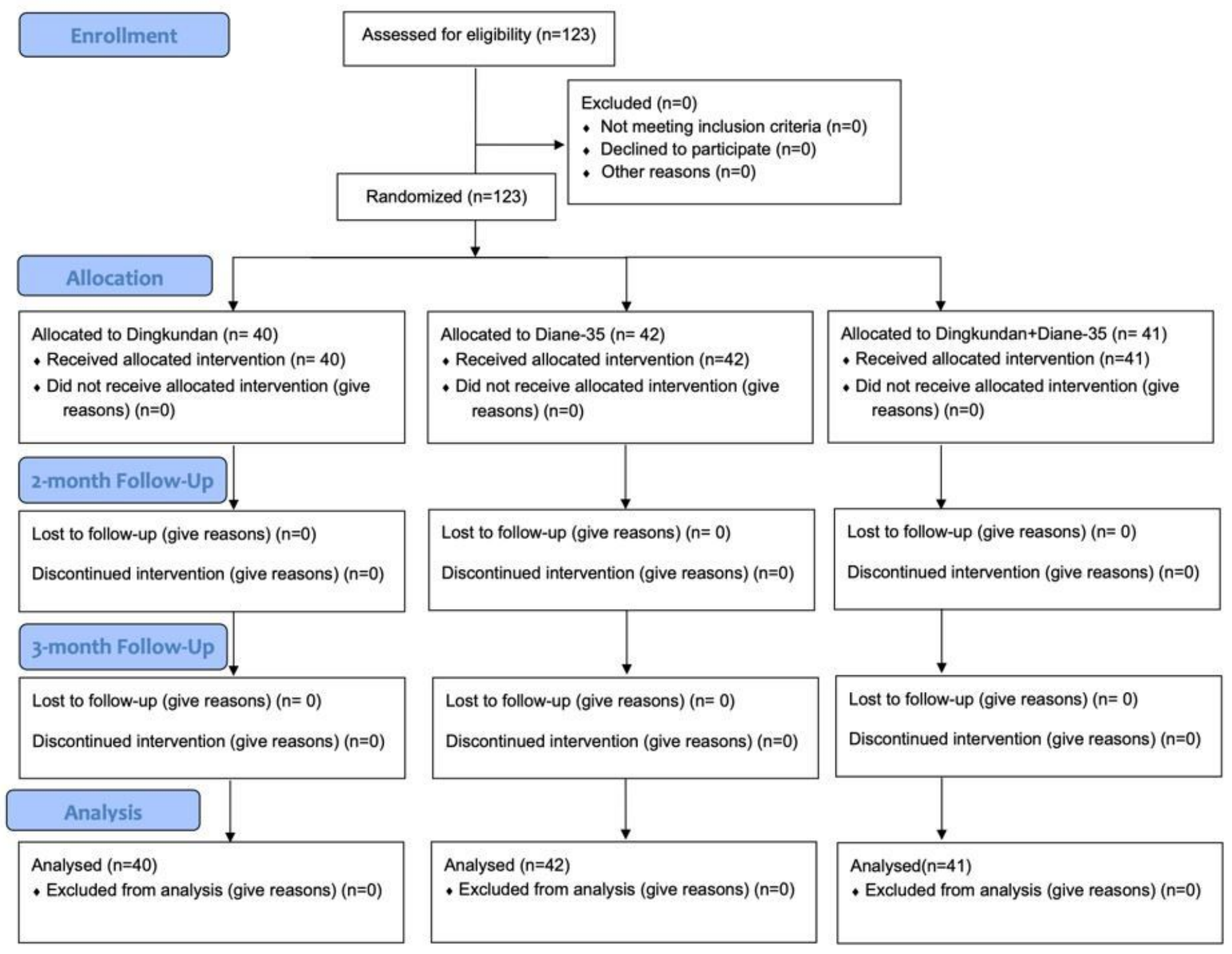

\section{Figure 1}

Flowchart of the study. 


\section{Metabolite Sets Enrichment Overview}

Malate-Aspartate Shuttle Aspartate Metabolism

Nicotinate and Nicotinamide Metabolism Urea Cycle Lysine Degradation Ammonia Recycling Citric Acid Cycle

Biotin Metabolism Amino Sugar Metabolism

Glutathione Metabolism

Phenylacetate Metabolism Glucose-Alanine Cycle

Phenylalanine and Tyrosine Metabolism Warburg Effect

Pyrimidine Metabolism

Purine Metabolism

Alanine Metabolism

Beta Oxidation of Very Long Chain Fatty Acids

Glutamate Metabolism

Beta-Alanine Metabolism

Catecholamine Biosynthesis

Carnitine Synthes is

Transfer of Acetyl Groups into Mitochondria

Tyrosine Metabolism

Androstenedione Metabolism

Glycine and Serine Metabolism

Histidine Metabolism

Glycerolipid Metabolism

Tryptophan Metabolism

Valine, Leucine and Isoleucine Degradation

Cysteine Metabolism

Oxidation of Branched Chain Fatty Acids

Steroid Biosynthesis

Folate Metabolism

Arginine and Proline Metabolism Androgen and Estrogen Metabolism

Retinol Metabolism

Porphyrin Metabolism

Propanoate Metabolism Arachidonic Acid Metabolism
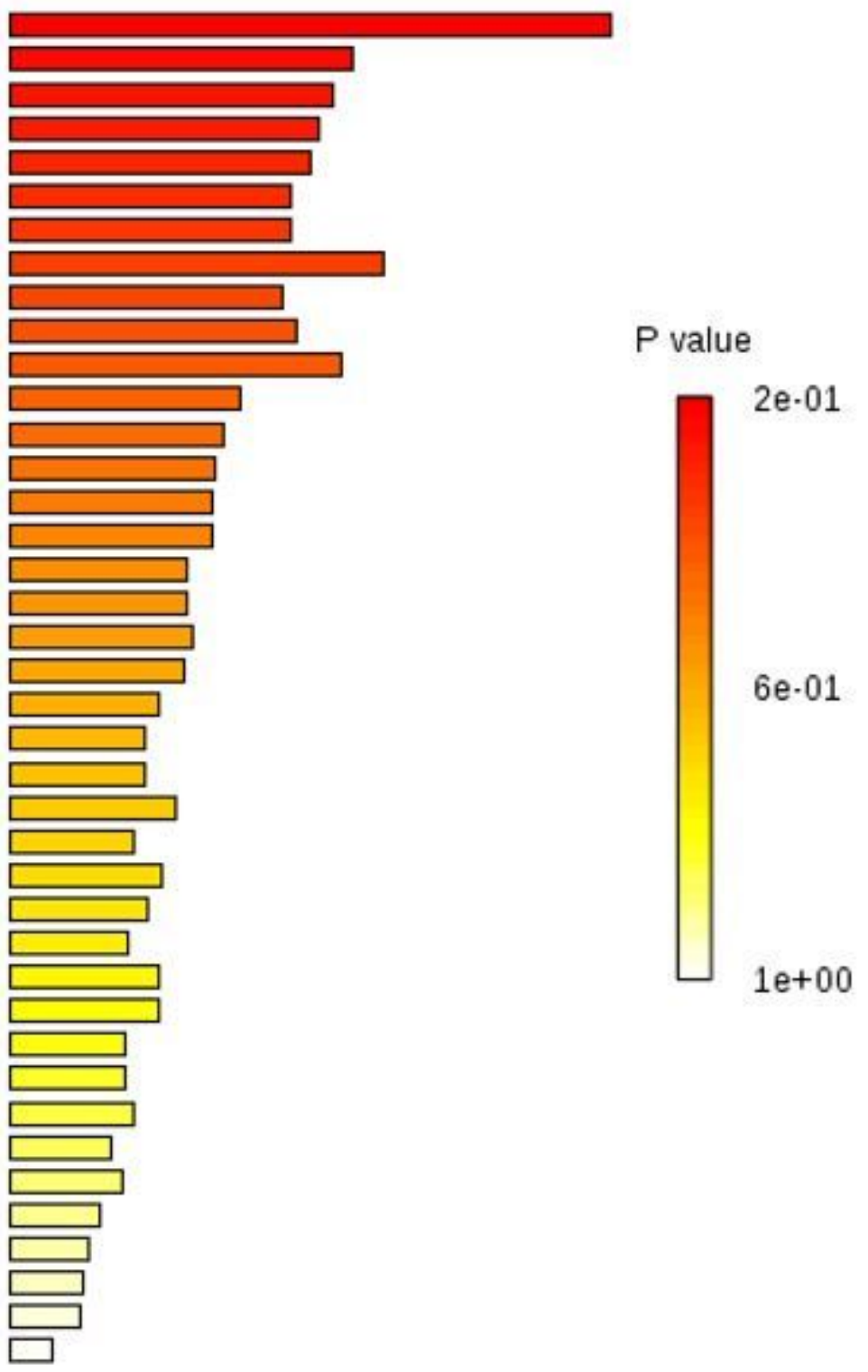

$1 e+00$

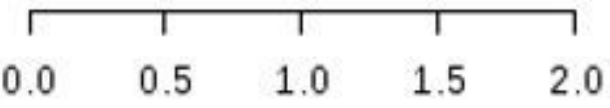

Fold Enrichment

Figure 2

The fold enrichment of metabolites. 
A.

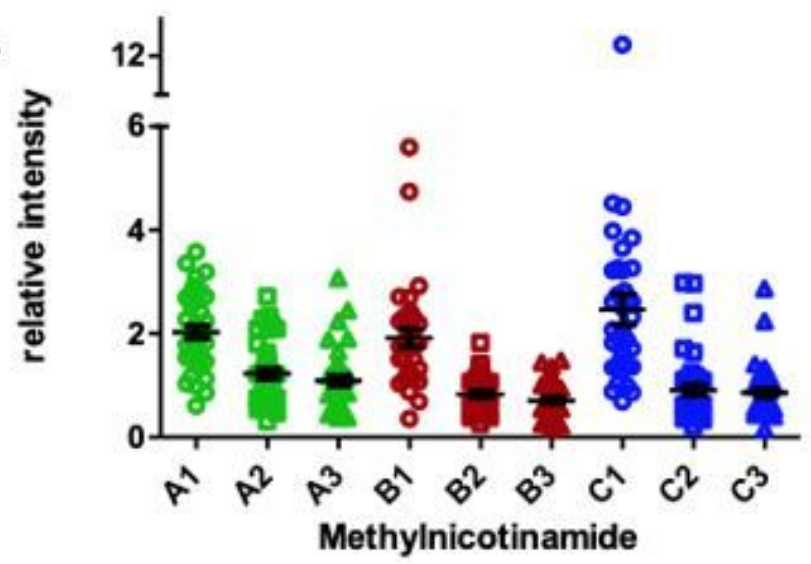

B.

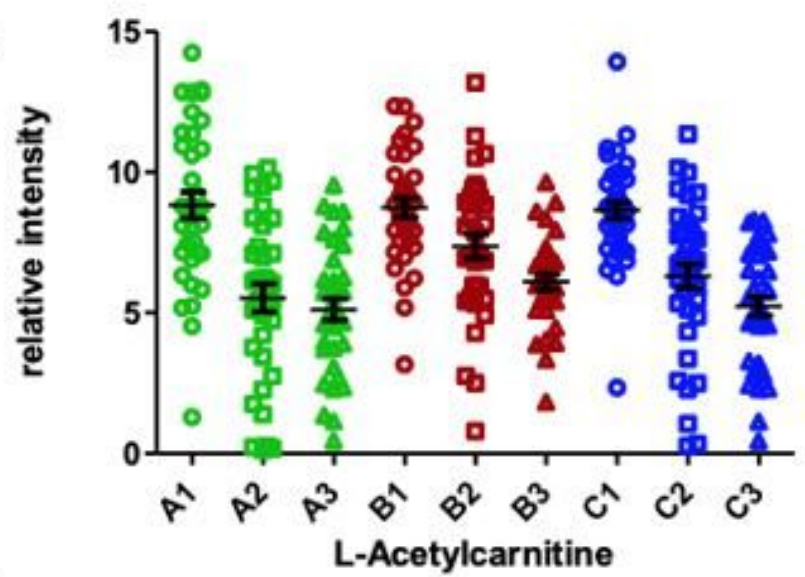

C.

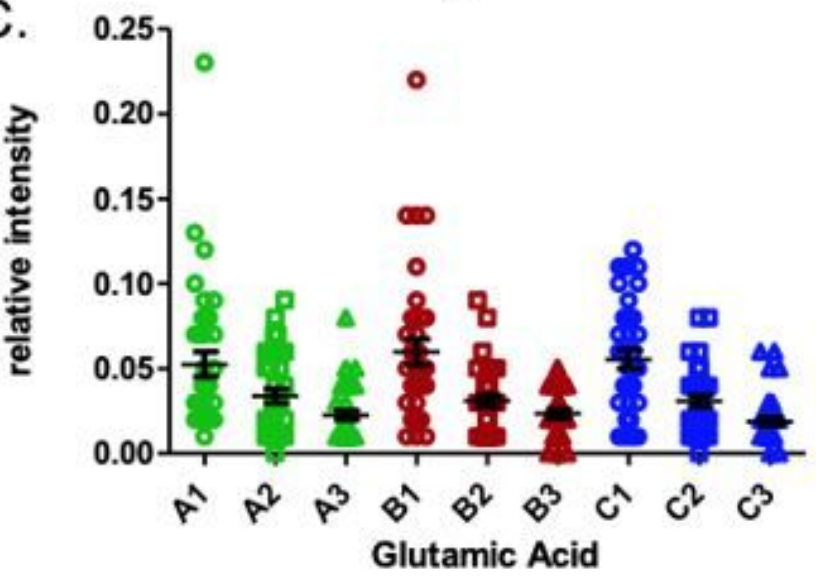

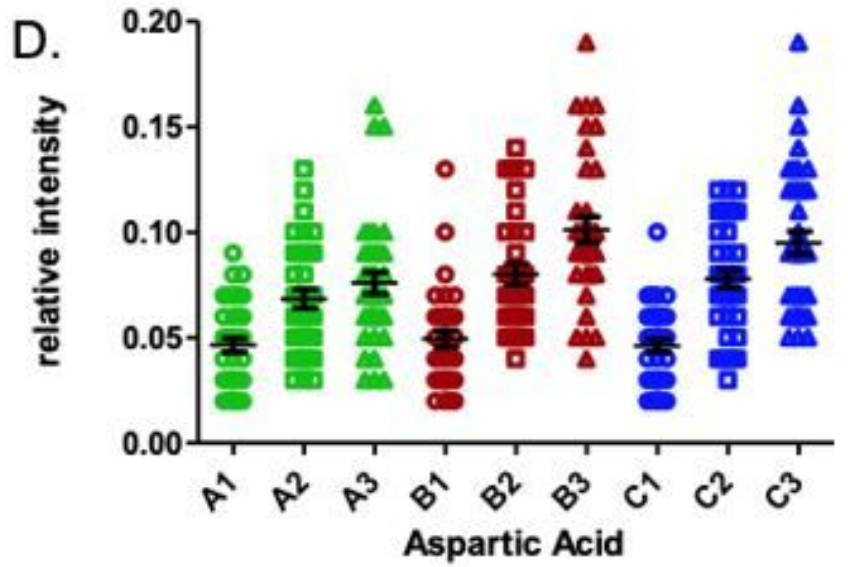

E.

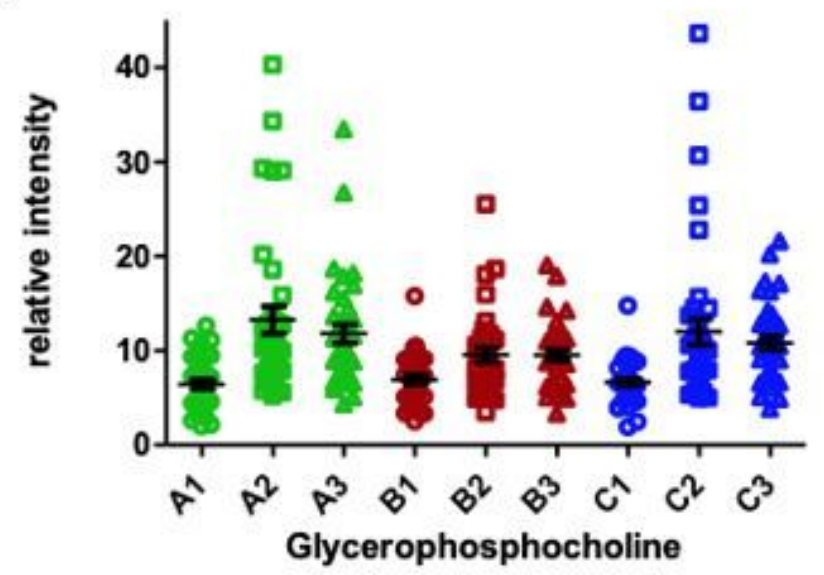

$\mathrm{F}$.

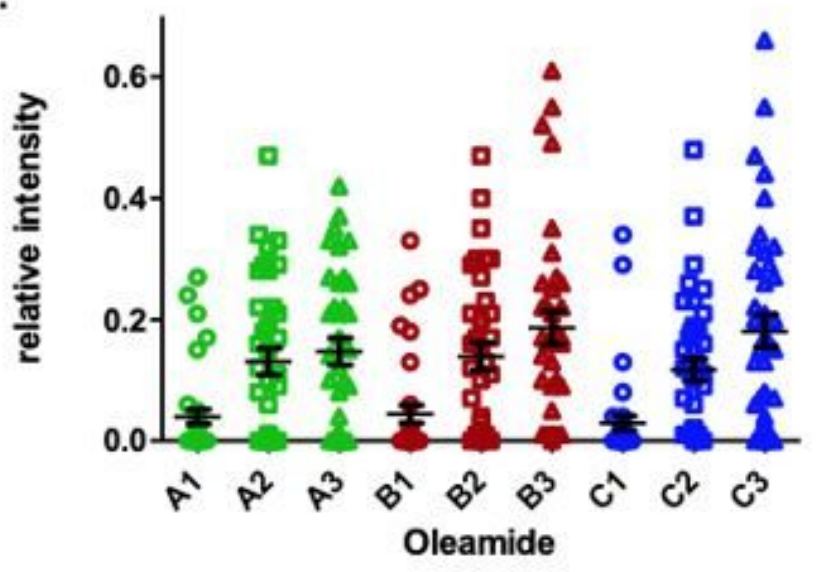

Figure 3

Abundance of the set of 6 metabolites after various medications. (A) methylnicotinamide, (B) acetylcarnitine, (C) glutamic acid, (D) aspartic acid, (E) glycerophosphocholine and (F) oleamide Group A1, B1, and C1 are from serums prior to drug treatment. Group A2, B2, and C2 as well as group A3, B3, and $\mathrm{C} 3$ are the ones after taking 2-month and 3-month medicine, respectively. 
A.

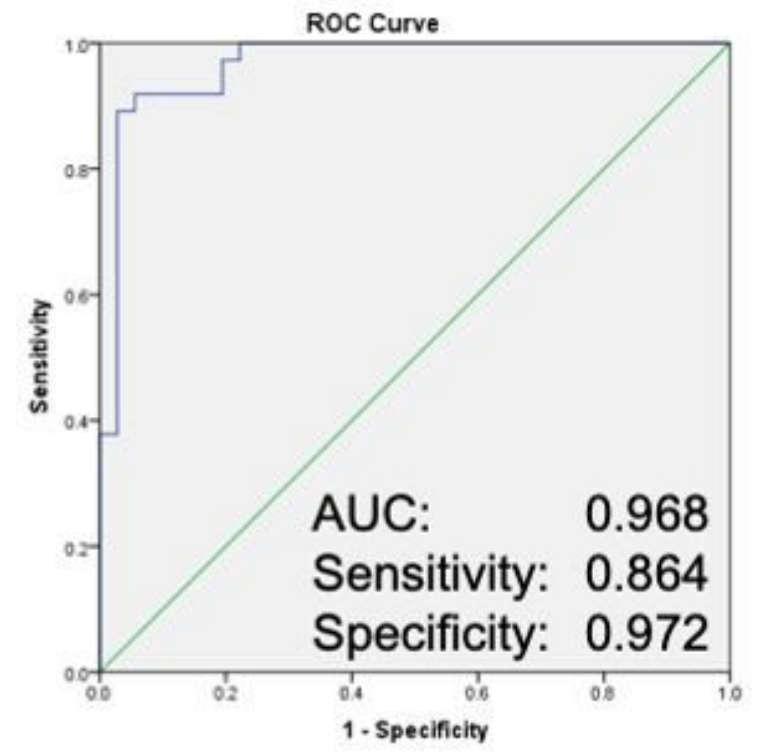

C.

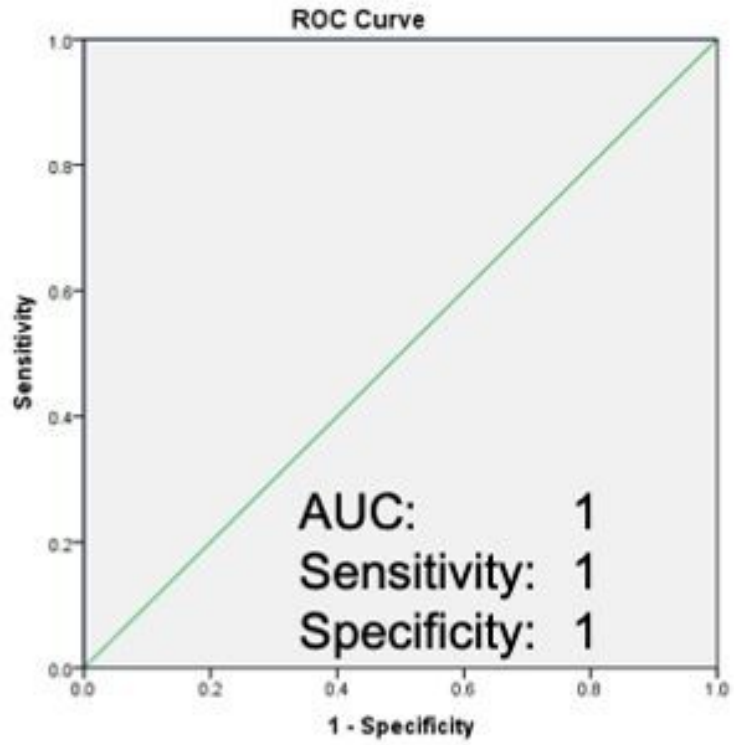

B.

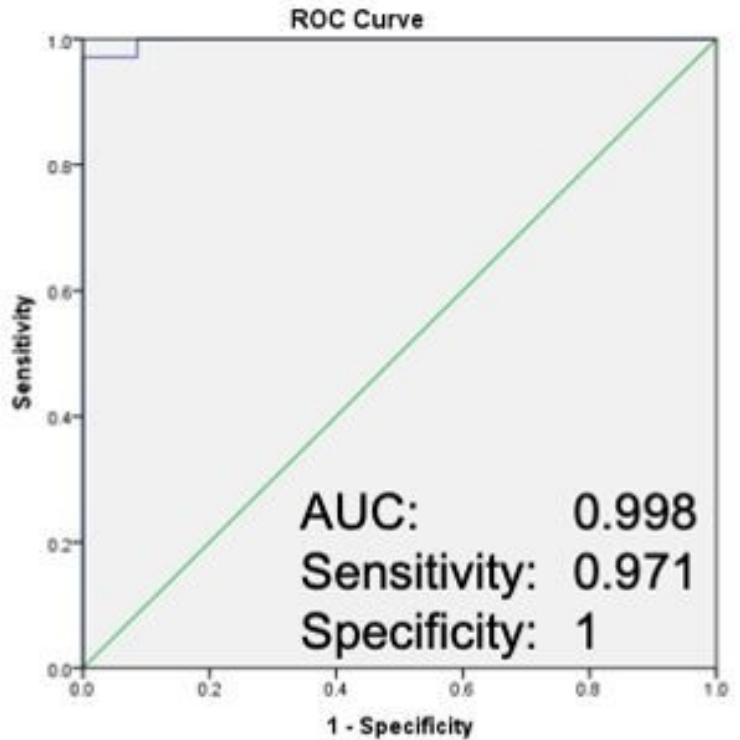

D.

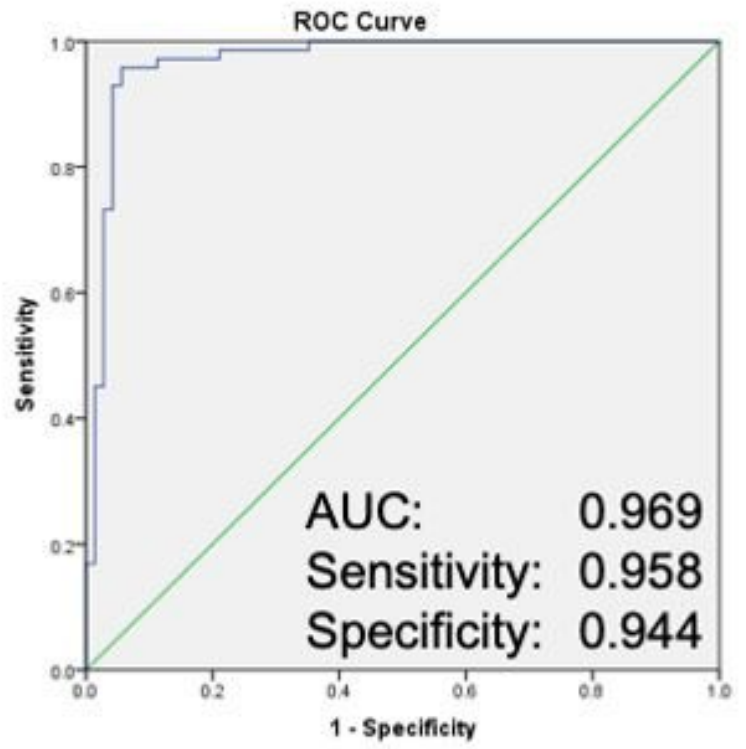

Figure 4

ROC curves of the set of 6 metabolites. Methylnicotinamide, acetylcarnitine, glutamic acid, aspartic acid, glycerophosphocholine, and oleamide were used to evaluate drug efficacy in the group of $(A)$ herbal medicine, (B) western drug, (C) combo drugs and (D) combined herbal and western drug groups. 


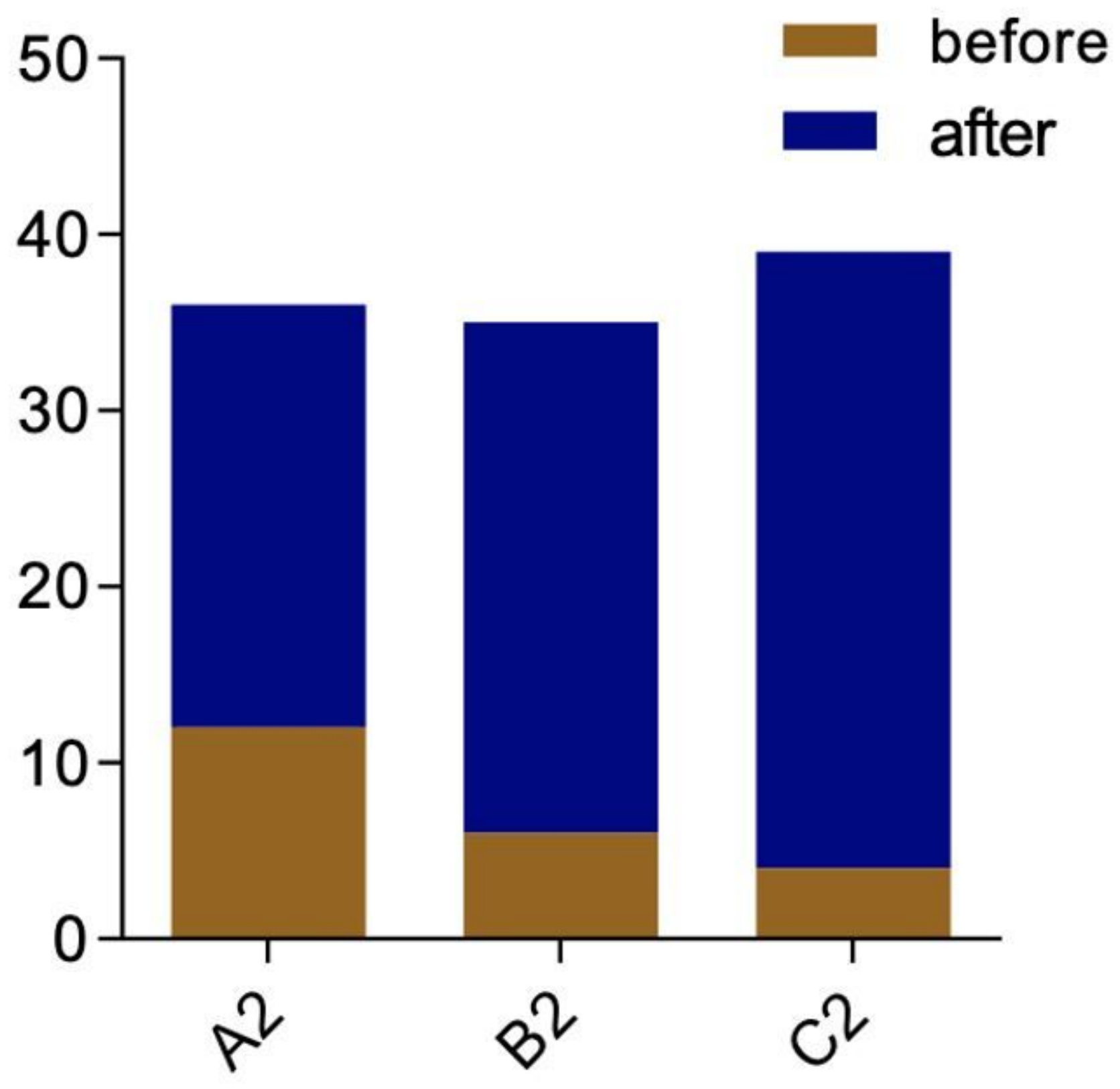

Figure 5

ROC curves of the set of 6 metabolites after two-month treatment. Methylnicotinamide, acetylcarnitine, glutamic acid, aspartic acid, glycerophosphocholine, and oleamide were used to evaluate drug effect in the group of $(A)$ herbal medicine, $(B)$ western drug, $(C)$ combo drugs after two-month treatment.

\section{Supplementary Files}

This is a list of supplementary files associated with this preprint. Click to download.

- CONSORT2010Checklist.pdf

- additionalfile.docx 
Page 20/20 